\title{
Inhibition of protein SUMOylation by davidiin, an ellagitannin from Davidia involucrata
}

\author{
Misao Takemoto ${ }^{1,2}$, Yumi Kawamura ${ }^{3}$, Mikako Hirohama ${ }^{4,5,6}$, Yuki Yamaguchi ${ }^{2}$, Hiroshi Handa ${ }^{7}$, \\ Hisato Saitoh ${ }^{8}$, Yoichi Nakao ${ }^{5}$, Manabu Kawada ${ }^{9}$, Khan Khalid ${ }^{4}$, Hiroyuki Koshino ${ }^{10}$, Ken-ichi Kimura ${ }^{3}$, \\ Akihiro Ito ${ }^{4,11}$ and Minoru Yoshida ${ }^{1,4,6,11}$
}

The Journal of Antibiotics (2014) 67, 335-338; doi:10.1038/ja.2013.142; published online 15 January 2014

Keywords: Davidia involucrata; Davidiin; ellagitannin; SUMOylation inhibitor

Conjugation of small ubiquitin-related modifier (SUMO) to lysine residues in target proteins is a multistep enzymatic reaction analogous to ubiquitination. $^{1}$ Protein SUMOylation regulates numerous biological processes including transcription, the cell cycle, DNA repair and innate immunity. ${ }^{1}$ In the first step of the reaction, SUMO is cleaved from the SUMO precursor by SUMO-specific proteases. Next, SUMO is bound to the cysteine residue of the SUMO-activating enzyme (E1), forming a thioester linkage in an ATP-dependent manner. SUMO is then transferred from E1 to the cysteine residue of the SUMO-conjugating enzyme (E2). Finally, SUMO ligase (E3) catalyzes the SUMOylation of specific substrates via a direct interaction with $\mathrm{E} 2$ and the substrates. Like ubiquitination, SUMOylation is reversible; the deSUMOylation process is mediated by SUMO-specific proteases. Abnormal SUMOylation is implicated in various diseases including neurodegenerative disease, ${ }^{2}$ viral infection ${ }^{3}$ and cancer. ${ }^{4,5}$ Therefore, enzymes responsible for the SUMO conjugation pathway represent potential targets for drug discovery.

To date, several natural products including ginkgolic acid, ${ }^{6}$ anacardic acid, ${ }^{6}$ kerriamycin $\mathrm{B}^{7}$ and spectomycin $\mathrm{B1}^{8}$ as well as synthetic compounds, ${ }^{9}$ have been reported to inhibit protein SUMOylation. Here, we report another natural product that functions as a SUMOylation inhibitor: davidiin, purified from the plant Davidia involucrata. Although most known SUMOylation inhibitors function in the micromolar range, davidiin is particularly potent, inhibiting at sub-micromolar concentrations.

Materials for this study were obtained as follows. Goat polyclonal anti-SUMO-1 (N-19) and goat polyclonal anti-p53 (FL393)-G antibodies were purchased from Santa Cruz Biotechnologies (Santa Cruz, CA, USA). A mouse monoclonal anti-T7 antibody was from Novagen
(Darmstadt, Germany). Mouse monoclonal anti- $\alpha$-tubulin (B-5-1-2) and anti-FLAG (M2) antibodies were purchased from Sigma (St. Louis, MO, USA). Recombinant His- and T7-tagged RanGAP1-C2, GST-Aos1-Uba2 fusion protein (E1), His-tagged Ubc9 (E2), and Histagged SUMO-1 proteins were purified as described previously. ${ }^{10}$ 293T, H1299, MKN-45, DU-145 and NCI-H460 cells were maintained in Dulbecco's modified Eagle medium supplemented with $10 \%$ FBS at $37^{\circ} \mathrm{C}$ under $5 \% \mathrm{CO}_{2}$.

The in vitro SUMOylation reaction was performed as described. ${ }^{6}$ Briefly, in vitro SUMOylation reaction was performed for $2 \mathrm{~h}$ at $30^{\circ} \mathrm{C}$ in $20 \mu$ l buffer ( $50 \mathrm{~mm}$ Tris- $\mathrm{HCl}$ (pH 7.4), $6 \mathrm{~mm} \mathrm{MgCl}_{2}, 2 \mathrm{~mm}$ ATP and $1 \mathrm{~mm}$ dithiothreitol) containing His- and T7-tagged RanGAP1-C2, GST-Aos1/Uba2 (E1), His-tagged Ubc9 and His-tagged SUMO-1. Samples were separated by $10 \%$ SDS-PAGE followed by immunoblotting using an anti-T7 antibody and an anti-SUMO-1 antibody.

The reaction for thioester bond formation between SUMO and E1 was performed as described. ${ }^{6}$ Briefly, the reaction for the thioester bond formation was performed for $20 \mathrm{~min}$ at $37^{\circ} \mathrm{C}$ in $20 \mu \mathrm{l}$ buffer (50 mм Tris- $\mathrm{HCl}$ ( $\mathrm{pH} 7.4$ ), 6 mм $\mathrm{MgCl}_{2}, 2$ mм ATP) containing GSTAos1/Uba2 (E1) and biotinylated SUMO-1 in the absence of dithiothreitol. Samples were separated by $11 \%$ SDS-PAGE and the E1-biotinylated SUMO-1 intermediate was detected by avidin-conjugated horseradish peroxidase (Sigma).

A screen of 750 samples of botanical and food ingredients extracts using an in situ cell-based SUMOylation assay ${ }^{11}$ revealed several samples that could inhibit protein SUMOylation, including an extract of D. involucrata (data not shown). ${ }^{6}$ The inhibitory activity of the D. involucrata extract was confirmed by in vitro SUMOylation assay using RanGAP1-C2 as substrate (Figure 1a). Compound A was isolated by activity-guided fractionation and it was identified by

${ }^{1}$ Drug Discovery Platform Cooperation Division, RIKEN Center for Sustainable Resource Science, Wako, Japan; ${ }^{2}$ Department of Biological Information, Tokyo Institute of Technology, Yokohama, Japan; ${ }^{3}$ Graduate School of Agriculture, Iwate University, Morioka, Japan; ${ }^{4}$ Chemical Genetics Laboratory, RIKEN, Wako, Japan; ${ }^{5}$ Department of Chemistry and Biochemistry, Waseda University, Tokyo, Japan; ${ }^{6}$ Japan Science and Technology Corporation, CREST Research Project, Kawaguchi, Japan; ${ }^{7}$ Graduate School of Bioscience and Biotechnology, Tokyo Institute of Technology, Yokohama, Japan; ${ }^{8}$ Department of New Frontier Sciences, Graduate School of Science and Technology, Kumamoto University, Kumamoto, Japan; 9 Institute of Microbial Chemistry (BIKAKEN), Numazu, Microbial Chemistry Research Foundation, Numazu, Japan; 10 Global Research Cluster, RIKEN, Wako, Japan and ${ }^{11}$ Chemical Genomics Research Group, RIKEN Center for Sustainable Resource Science, Wako, Japan

Correspondence: Dr A Ito, Chemical Genetics Laboratory, RIKEN, 2-1 Hirosawa, Wako 351-0198, Japan.

E-mail: akihiro-i@riken.jp

Received 27 September 2013; revised 22 November 2013; accepted 25 November 2013; published online 15 January 2014 


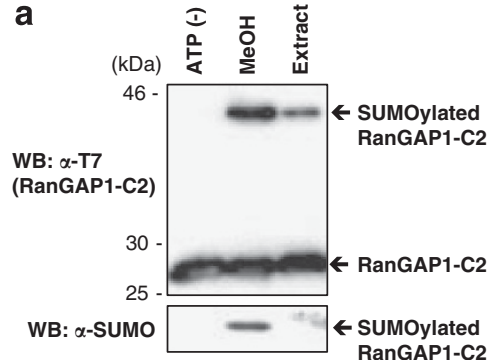

b
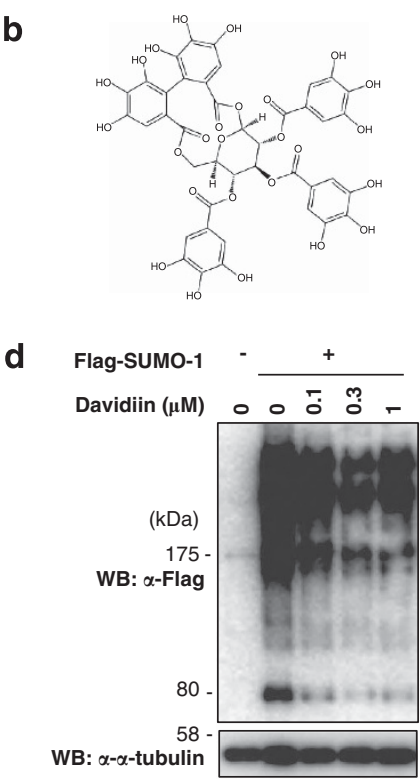

f

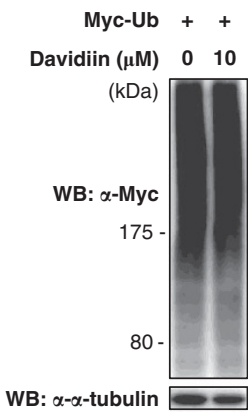

C
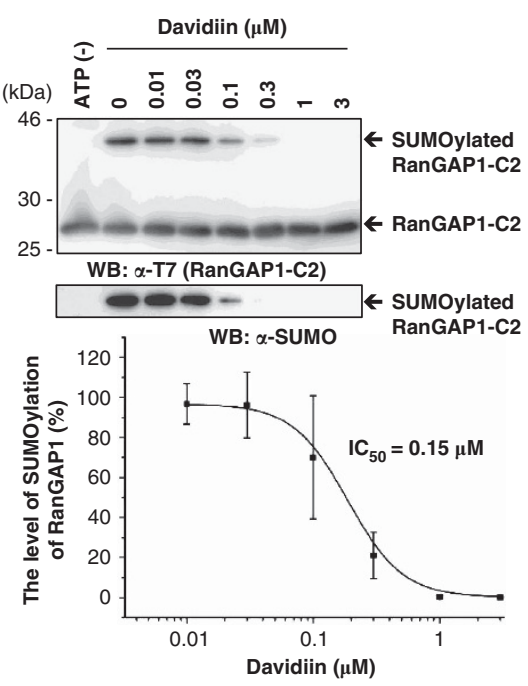

$\begin{array}{rrrrrr}\text { GFP-SUMO-1 } & - & + & + & + \\ \text { p53 wt } & + & + & + & + \\ \text { Davidiin }(\mu \mathrm{M}) & 0 & 0 & 1 & 10\end{array}$
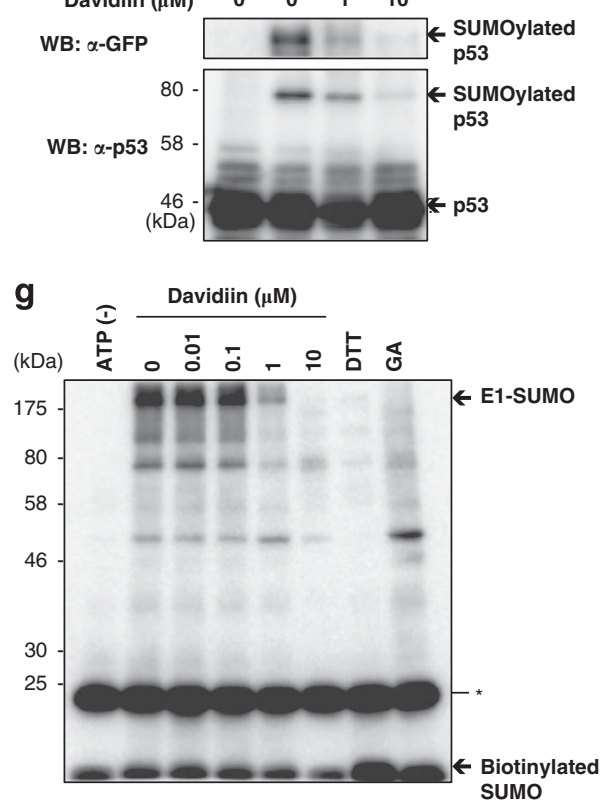

Figure 1 Davidiin inhibits protein SUMOylation. (a) Inhibition of protein SUMOylation by $\mathrm{MeOH}$ extracts of Davidia involucrata. D. involucrata extract $\left(1 \mathrm{~g} \mathrm{ml}^{-1}\right)$ was added to a SUMOylation reaction mixture and SUMOylated RanGAP1-C2 was detected by immunoblotting using an anti-T7 or anti-SUMO-1 antibody. (b) Structure of davidiin. (c) Dose response of davidiin for SUMOylation inhibition. SUMOylated RanGAP1-C2 was detected as described in a. The intensity of SUMOylated RanGAP1 was measured using an Image Gauge Version 4.22 (Fujifilm). Error bars show the standard deviations from three independent assays, and the $\mathrm{IC}_{50}$ value was calculated. (d) Inhibition of in vivo SUMOylation by davidiin. 293T cells expressing FLAG-tagged SUMO-1 were treated with the indicated concentrations of davidiin for $12 \mathrm{~h}$ and cell lysates were separated by $6 \%$ SDS-PAGE, followed by immunoblotting using antiFLAG or anti- $\alpha$-tubulin antibody. (e) Effects of davidiin on p53 SUMOylation. H1299 cells (p53 ${ }^{-/}$) were transfected with p53 alone or with GFP-fused SUMO-1 and then treated with davidiin for $24 \mathrm{~h}$. The immune complex obtained with an anti-p53 (FL393)-G antibody was analyzed by immunoblotting using an anti-GFP or an anti-p53 (Ab-6) antibody. (f) Effects of davidiin on in vivo ubiquitination. 293T cells expressing Myc-tagged ubiquitin were treated with $10 \mu \mathrm{m}$ of davidiin for $12 \mathrm{~h}$ in the presence of $10 \mu \mathrm{m}$ of the proteasome inhibitor MG132. Cell lysates were separated by $6 \%$ SDS-PAGE, followed by immunoblotting using an anti-Myc antibody. (g) Inhibition of the thioester bond formation between E1 and biotinylated SUMO-1 by davidiin. Addition of $1 \mathrm{~mm}$ dithiothreitol or ginkgolic acid $(\mathrm{GA}, 10 \mu \mathrm{m})$ as a positive control ${ }^{6}$ abolished the formation of the E1-biotinylated SUMO-1 intermediate. The asterisk represents a non-specific band.

mass spectrometry (MS) (JMS-700, JEOL Ltd, Tokyo, Japan), NMR (EX-400, JEOL Ltd), UV (UV mini 1240, Shimadzu Co., Kyoto, Japan), and optical rotation (DIP1000, JASCO Co., Tokyo, Japan). Briefly, leaves of D. involucrata (328.3 g) were harvested from the Botanical Gardens of Iwate University and dried naturally at room temperature. The dried leaves $(113.0 \mathrm{~g})$ were extracted with $\mathrm{MeOH}$, and the extract was diluted with water. The $\mathrm{MeOH}$ extract $(34.05 \mathrm{~g})$ was extracted with ethyl acetate (1 vol, two times) and evaporated $(8.96 \mathrm{~g})$. Half of the ethyl acetate extract was subjected to a Diaion HP-20 column $(3 \mathrm{~cm}$ diameter $\times 20 \mathrm{~cm})$ and an active fraction was 
eluted with $50 \% \mathrm{MeOH}$ two times repeatedly ( $1.06 \mathrm{~g}$ ). Biologically active peak A was isolated from part of the $50 \% \mathrm{MeOH}$ fraction (100.0 mg) using an HPLC system (880-PU (pump) equipped with an MD-910 photodiode array detector (JASCO, Tokyo, Japan)) on a Capcell Pak ODS ( $20 \mathrm{~mm}$ diameter $\times 250 \mathrm{~mm}$; Shiseido Inc., Tokyo, Japan) with $15 \% \mathrm{CH}_{3} \mathrm{CN}-0.1 \% \mathrm{CH}_{3} \mathrm{COOH}$ at a flow rate of $5 \mathrm{ml} \mathrm{min}{ }^{-1}$. After the peak A fraction was isolated, it was subjected to $\mathrm{HP}-20$ column $(2.1 \mathrm{~cm}$ diameter $\times 7.0 \mathrm{~cm})$ and eluted with $50 \%$ $\mathrm{MeOH}$. The product was obtained as a light brownish powder after lyophilization $(18.0 \mathrm{mg})$.

Data obtained were as follows: HR-FAB-MS $m / z(M+\mathrm{H})^{+}$, calculated for $\mathrm{C}_{41} \mathrm{H}_{31} \mathrm{O}_{26}, 939.1104$; found, 939.1152; ${ }^{1} \mathrm{H}$ NMR $\delta_{\mathrm{H}}$ of the glucose moiety of compound $\mathrm{A}\left(400 \mathrm{MHz}\right.$, acetone- $\left.\mathrm{d}_{6}\right) 6.13$ $(1 \mathrm{H}, \mathrm{d}, J=2.7), 5.53(1 \mathrm{H}, \mathrm{dd}, J=2.7,7.0), 5.79(1 \mathrm{H}, \mathrm{dd}, J=6.5,7.0)$, $5.21(1 \mathrm{H}, \mathrm{dd}, J=2.7,6.5), 4.57(1 \mathrm{H}, \mathrm{ddd}, J=2.7,5.1,12.1), 4.84(1 \mathrm{H}$, $\mathrm{dd}, J=11.7,12.1), 4.42(1 \mathrm{H}, \mathrm{dd}, J=5.1,11.7),{ }^{13} \mathrm{C} \quad \mathrm{NMR} \delta_{\mathrm{C}}$ (100 MHz, acetone- $\mathrm{d}_{6}$ ) 94,1 (C-1), 70.2 (C-2), 68.7 (C-3), 70.7 (C4), 74.9 (C-5), 64.8 (C-6); the UV spectrum (nm) ( $\varepsilon$ ) in $\mathrm{MeOH} 279$ (49800); $[\alpha]_{\mathrm{D}}^{25}=+20.2^{\circ} \quad(c=0.2, \mathrm{MeOH})$. All physicochemical properties were identical with data reported for a known ellagitannin, davidiin, previously isolated from $D$. involucrata (Figure 1b). ${ }^{12-14}$ Purified davidiin inhibited in vitro SUMOylation of RanGAP1-C2 in a dose-dependent manner, and its $\mathrm{IC}_{50}$ value was $0.15 \mu \mathrm{M}$ (Figure $1 \mathrm{c}$ ). We next examined whether davidiin affects in vivo protein SUMOylation by analyzing the levels of SUMOylated proteins in 293T cells expressing FLAG-tagged SUMO-1. Immunoblotting using an antiFLAG antibody revealed that davidiin reduced the amount of highmolecular weight SUMO conjugates in a dose-dependent manner (Figure 1d). In addition, davidiin reduced the level of SUMOylation of p53 in a dose-dependent manner (Figure 1e). Importantly, davidiin did not influence the cellular level of ubiquitinated proteins (Figure 1f). These results indicate that davidiin inhibits protein SUMOylation both in vitro and in vivo without affecting protein ubiquitination. Next, we sought to identify the target of davidiin. The formation of an E1 conjugate to biotinylated SUMO-1 via a thioester linkage could be detected in the presence of ATP under non-reducing conditions; the band corresponding to the E1biotinylated SUMO-1 intermediate disappeared when the reducing agent dithiothreitol or the SUMO E1 inhibitor ginkgolic acid ${ }^{6}$ was added to the reaction mixture (Figure $1 \mathrm{~g}$ ). The formation of an intermediate between E1 and biotinylated SUMO-1 was also blocked by davidiin in a dose range similar to that required for inhibition of in vitro SUMOylation (Figures $1 \mathrm{c}$ and $\mathrm{g}$ ). These results suggest that davidiin inhibits protein SUMOylation by inhibiting the formation of the E1-SUMO-1 intermediate.

High-level expression of genes involved in SUMOylation was frequently observed in cancer. In addition, SUMO E1 has an important role in Myc-driven tumorigenesis. ${ }^{15}$ Accordingly, we tested effects of davidiin on proliferation of cancer cells. As shown in Figure 2, davidiin inhibited cell proliferation of several cancer cells in a dose-dependent manner with similar $\mathrm{GI}_{50}$ values (gastric cancer MKN-45 cells: $8.3 \mu \mathrm{M}$, prostate cancer DU-145 cells: $11.6 \mu \mathrm{M}$, lung cancer NCI-H460 cells: $16.4 \mu \mathrm{M}$ ).

Davidiin is an ellagitannin previously shown to inhibit the binding of a ligand to a $\mu$-opioid receptor. ${ }^{16}$ In this study, we demonstrated a novel physiological activity of davidiin, namely, inhibition of protein SUMOylation. The $\mathrm{IC}_{50}$ value of davidiin against in vitro protein SUMOylation is $0.15 \mu \mathrm{M}$ (Figure 1c), the most potent among smallmolecule SUMOylation inhibitors reported to date. ${ }^{6-9}$ Recent studies have shown that davidiin inhibits growth of hepatocellular carcinoma cells by downregulating $\mathrm{EZH} 2,{ }^{17}$ the enzymatic subunit of the

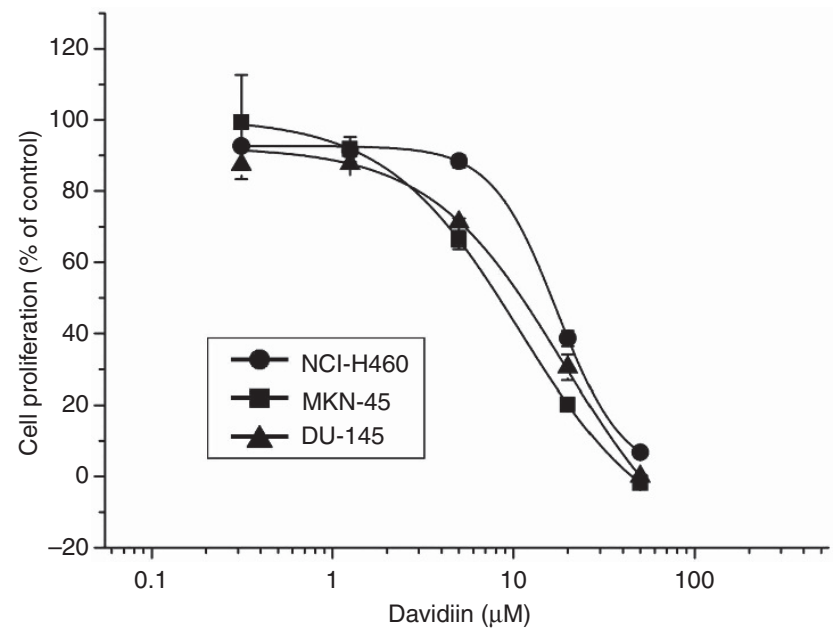

Figure 2 Davidiin inhibits cancer cells growth. Cells were treated with davidiin at various concentrations ranged from 0.3 to $50 \mu \mathrm{m}$ for $96 \mathrm{~h}$. Effects on cell proliferation were evaluated by WST-1 assays.

Polycomb-repressive complex 2 that catalyzes histone H3 lysine 27 methylation, which is highly expressed in a variety of human cancers. Importantly in this regard, EZH2 is SUMOylated in cells. ${ }^{18}$ In this study, we showed that davidiin inhibited cell growth of several cancer cells including DU-145 cells, in which EZH2 is overexpressed (Figure 2). ${ }^{19}$ Importantly, concentrations of $\mathrm{GI}_{50}$ values of davidiin for these cancer cells were sufficient to remarkably inhibit protein SUMOylation in cells (Figures $1 \mathrm{~d}$ and e). Although the molecular mechanism by which davidiin inhibits cancer cell growth remains to be elucidated, these observations suggested that the anti-tumor activity of davidiin is mediated, at least in part, by inhibition of SUMOylation of proteins including EZH2. The total synthesis of davidiin was recently established by Kasai et al. ${ }^{20}$ Because davidiin is the strongest natural SUMOylation inhibitor reported so far, it is an excellent starting material for drug discovery. Analyses of the structure-activity relationship of this compound should facilitate development of novel anticancer agents targeting protein SUMOylation.

\section{ACKNOWLEDGEMENTS}

This work was supported in part by the Chemical Genomics Research Project; RIKEN Center for Sustainable Resource Science; the CREST Research Project; the Japan Science and Technology Corporation; Grants-in-Aid for Scientific Research (C); and Grants-in-Aid for Scientific Research on Innovative Area 'Cancer' from the Ministry of Education, Culture, Sports, Science and Technology of Japan.

1 Johnson, E. S. Protein modification by SUMO. Annu. Rev. Biochem. 73, 355-382 (2004).

2 Dorval, V. \& Fraser, P. E. SUMO on the road to neurodegeneration. Biochim. Biophys. Acta 1773, 694-706 (2007).

3 Boggio, R. \& Chiocca, S. Viruses and sumoylation: recent highlights. Curr. Opin. Microbiol. 9, 430-436 (2006).

4 Alarcon-Vargas, D. \& Ronai, Z. SUMO in cancer-wrestlers wanted. Cancer Biol. Ther. 1, 237-242 (2002)

5 Moschos, S. J. \& Mo, Y. Y. Role of SUMO/Ubc9 in DNA damage repair and tumorigenesis. J. Mol. Histol. 37, 309-319 (2006).

6 Fukuda, I. et al. Ginkgolic acid inhibits protein SUMOylation by blocking formation of the E1-SUMO intermediate. Chem. Biol. 16, 133-140 (2009).

7 Fukuda, I. et al. Kerriamycin B inhibits protein SuMOylation. J. Antibiot. 62, 221-224 (2009). 
8 Hirohama, M. et al. Spectomycin B1 as a Novel SUMOylation Inhibitor That Directly Binds to SUMO E2. ACS Chem. Biol. 8, 2635-2642 (2013).

9 Kim, Y. S., Nagy, K., Keyser, S. \& Schneekloth, J. S. Jr. An electrophoretic mobility shift assay identifies a mechanistically unique inhibitor of protein sumoylation. Chem. Biol. 20, 604-613 (2013)

10 Uchimura, Y., Nakao, M. \& Saitoh, H. Generation of SUMO-1 modified proteins in E. coli: towards understanding the biochemistry/structural biology of the SUMO-1 pathway. FEBS Lett. 564, 85-90 (2004).

11 Saitoh, N. et al. In situ SUMOylation analysis reveals a modulatory role of RanBP2 in the nuclear rim and PML bodies. Exp. Cell Res. 312, 1418-1430 (2006).

12 Haddock, E. A., Gupta, R. K. \& Haslam, E. The metabolism of gallic acid and hexahydroxydiphenic acid in plants. Part 3. Esters of (R)- and (S)-hexahydroxydiphenic acid and dehydrohexahydroxydiphenic acid with D-glucopyranose $\left({ }^{1} \mathrm{C}_{4}\right.$ and related conformations). J. Chem. Soc. Perkin Trans. 1 1, 2535-2545 (1982).

13 Hatano, T., Hattori, S., Ikeda, Y., Shingu, T. \& Okuda, T. Gallotannins Having a 1, 5-Anhydro-D-glucitol Core and Some Ellagitannins from Acer Species. Chem. Pharm. Bull. 38, 1902-1905 (1990).
14 Yoshida, T. et al. Tannins of Euphorbiaceous Plants. X. Antidesmin A, a New Dimeric Hydrolyzable Tannin from Antidesma pentandrum var. barbatum. Chem. Pharm. Bull 40, 338-342 (1992).

15 Kessler, J. D. et al. A SUMOylation-dependent transcriptional subprogram is required for Myc-driven tumorigenesis. Science 335, 348-353 (2012).

16 Zhu, M., Phillipson, J. D., Greengrass, P. M., Bowery, N. E. \& Cai, Y. Plant polyphenols: biologically active compounds or non-selective binders to protein? Phytochemistry 44, 441-447 (1997).

17 Wang, Y. et al. A potential antitumor ellagitannin, davidiin, inhibited hepatocellular tumor growth by targeting EZH2. Tumour. Biol. doi:10.1007/s13277-013-1025-3 (2013).

18 Riising, E. M., Boggio, R., Chiocca, S., Helin, K. \& Pasini, D. The polycomb repressive complex 2 is a potential target of SUMO modifications. PLoS One 3, e2704 (2008).

19 Shin, Y. J. \& Kim, J. H. The role of EZH2 in the regulation of the activity of matrix metalloproteinases in prostate cancer cells. PLoS One 7, e30393 (2012).

20 Kasai, Y., Michihata, N., Nishimura, H., Hirokane, T. \& Yamada, H. Total synthesis of (+)-davidiin. Angew. Chem. Int. Ed. Engl. 51, 8026-8029 (2012). 